\title{
La topología como acceso a la teoría lacaniana
}

\author{
Topology as Access to Lacanian Theory \\ Pablo Bernardo Sánchez Gómez \\ UNED (España)
}

Recibido: 12.04 .2020

Aceptado: 01.09.2020

\section{RESUMEN}

En este texto se lleva una lectura de la obra lacaniana a través del estudio de sus figuras topológicas. En este sentido, se afirma que el psicoanálisis lacaniano no hace uso de la topología como si no de una herramienta externa si tratase, es decir, como un modo a través del cual obtener ejemplos ilustrativos y explicaciones, sino que el propio pensamiento lacaniano es en sí mismo topológico y, por tanto, no puede ser comprendido al margen de esta dimensión que atraviesa la totalidad de su recorrido. Para ello se estudiarán seis figuras: el grafo del deseo, la cinta de Moebius, el toro, la botella de Klein, la esfera provista de un cross-cap y el nudo borromeo.

PALABRAS CLAVE
PSICOANÁLISIS, LACAN, TOPOLOGÍA, NUDO BORROMEO, DESEO.

\section{ABSTRACT}

This paper carries out a reading of Lacan's work by the study of its topological figures. Thus, it is stated that Lacanian Psychoanalisis does not take Topology as an external tool in order to bring some exemples and explanations; rather, Lacanian thought is itself topological and therefore it cannot be understood aside from this dimension which goes through all its path. In order to do so, we will study six figures: the graph of desire, Moebius strip, the torus, Klein bottle, the sphere with a cross-cap and the Borromean ring.

KEYWORDS

PSYCHOANALISIS, LACAN, TOPOLOGY, BORROMEAN RING, DESIRE. 


\section{INTRODUCCIÓN.}

CUANDO SE TRATA DE ESTUdiAR LA TOPOlOGÍA LACANIANA resulta necesario comprender, por una parte, como señala Miller, que ésta no constituye una disciplina autónoma e independiente dentro del corpus lacaniano que podría ser extirpada y desarrollada escolásticamente como si de una ciencia independiente se tratase (Miller, 2003: 34). En este sentido, no podría afirmarse que hay algo así como la topología lacaniana sino que, por el contrario, hay un pensamiento topológico en Lacan, un proceder que no puede ser comprendido sino desde las figuras y los cuerpos propios de la topología matemática. Ahora bien, al mismo tiempo, debe evitarse pensar que Lacan emplea la topología como una herramienta, esto es, que se limitaría a aplicar una disciplina ya consolidada a su teoría con vistas a ejemplificar e ilustrar de forma intuitiva un pensamiento de alta complejidad y abstracción como es la clínica lacaniana. En este sentido, como señalan Triska y D'agord, «tomando la topología como guía expositiva estaríamos sirviéndonos de la intuición espacial euclidiana, lo que correspondería, en el campo de la clínica psicoanalítica, a un estudio orientado por lo imaginario» (Couto, 2013: 152) y, por tanto, estaríamos atendiendo exclusivamente a una de las tres dimensiones en la teoría psicoanalítica lacaniana. De este modo, se acepta la propuesta de Nasio al señalar que «para trazar una línea de demarcación entre la topología clásica y la nuestra habría que proceder como en el caso de la lingüística e inventar un nombre, por ejemplo topologería» (Nasio, 2007: 10).

De lo que se trata en este momento, por tanto, es de definir el objeto propio de estudio de la topologería. En este sentido, se acepta aquí la afirmación de Hoens cuando señala que la topología irrumpe en Lacan, no como suele afirmarse, quizá algo irreflexivamente, en el seminario XX sino que, por el contrario, se encuentran ya presentes cuerpos y objetos estudiados topológicamente desde sus primeros seminarios (Hoens, 2016: 56). De este modo, si bien la propuesta de Miller es atender a dos «estadios» o «etapas» dentro de la topologería lacaniana, el primero de ellos dedicado a la topología de superficies (esto es, a la cinta de Moebius, la botella de Klein y la esfera provista de un cross-cap) y el segundo a la topología de los nudos (Miller, 2003: 34); y, por otra parte, sin rechazar la clasificación de Nasio (Nasio, 2007: 13) y Monteiro (Monteiro, 2014: 134) donde se establecen únicamente cuatro objetos topológicos (el toro, 
la cinta de Moebius, la botella de Klein y la esfera provista de un crosscap), en el presente estudio se acepta el enfoque de Cao al entender que la topología, y con ella la topologería, se encarga simplemente del estudio de espacios en continua deformación, cambio y transición, para lo que emplea únicamente las nociones de "punto» y «proximidad» (Cao, S. F.: 13-14). Así, además de los cuerpos topológicos anteriormente citados, en este texto se comprenderá como objeto de estudio de la topologería el "grafo del deseo", entendiendo que el resto de cuerpos topológicos no son sino detalles, ampliaciones o desarrollos de lo contenido, potencialmente o de forma implícita, en el grafo.

De este modo, este texto se divide en tres secciones. En un primer momento, se introduce el grafo del deseo y se desarrolla su construcción y dinámica. Una vez finalizado este ejercicio, se introducen los cuatro objetos de superficies (cinta de Moebius, toro, botella de Klein y esfera provista de un cross-cap) para, en último lugar, introducir la importancia del nudo borromeo en la última enseñanza de Lacan.

\section{El GRAFO DEL DESEO: PRIMERA TOPOLOGÍA CONSOLIDADA.}

El grafo del deseo constituye el primer y fundamental esquema dinámico topológico de la enseńanza lacaniana y, por tanto, es la piedra fundamental de la topologería. En este sentido, si bien el grafo constituye en sí mismo un objeto definido y completo, no es menos cierto que es posible estudiar su funcionamiento a través de una serie de descomposiciones. En este sentido, el primer paso para comprender el mecanismo del grafo, y con él la práctica totalidad de la enseñanza lacaniana, consiste en señalar el primero de sus niveles, esto es, el piso inferior:

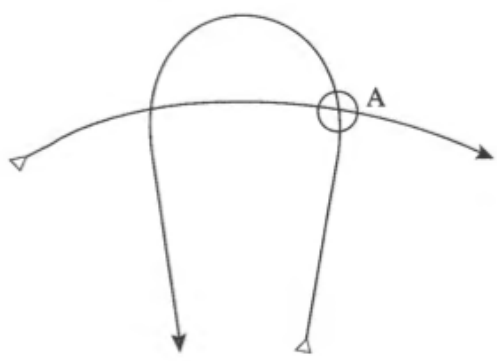

Imagen 1. La cadena significante. (Lacan, 2010: 16) 
En esta sencilla figura Lacan indica que la demanda, situada en el triángulo blanco inferior derecho, en la medida en que es la expresión de una necesidad inarticulada y como tal incondicional, atraviesa la cadena significante (compuesta por los extremos superiores izquierdo y derecho) y en este sentido figura, es decir, alcanza al Otro (representado por el punto "A», en la intersección resaltada con un círculo), lo que Lacan denominará «el tesoro del significante», siendo este Otro por tanto quien le entregará su necesidad ya conformada, esto es, constituida en una "demanda" (flecha inferior izquierda) (Lacan, 2010: 72-73). De este modo, Lacan concluye: «¿qué es una demanda? Es lo que de una necesidad, por medio del significante dirigido al Otro, pasa» (Lacan, 2010: 90). En este sentido, el movimiento necesidad-demanda, a través de la cadena significante, constituye un efecto retroactivo, après-coup, en el que es el Otro quien entrega al sujeto su demanda ya conformada. Ahora bien, esto es aceptar que la demanda del sujeto nunca se corresponde con su necesidad, que su necesidad queda necesariamente insatisfecha en cuanto demanda, ya que es el Otro, propiamente, quien demanda al sujeto, quien le da una demanda, su demanda más propia. En este sentido, como afirma Lacan,

al añadir el significante [a la necesidad] se le aporta un mínimo de transformación — de metáfora, por decirlo todo— que hace que lo significado sea algo más allá de la necesidad bruta, resulta remodelado por el uso del significante. En consecuencia, desde este comienzo, lo que entra en la creación del significado no es pura y simple traducción de la necesidad sino recuperación, reasunción, remodelado de la necesidad, creación de un deseo distinto de la necesidad. Es la necesidad más el significante (Lacan, 2010: 95).

Es en este momento cuando se presenta el grafo completo. Ahora bien, antes de tratarlo, resulta necesario comprender que esta "primera topología» o este primer estadio de la topologería no sería posible sin atender a la relación capital que Lacan constituye entre el sujeto y el significante. En este sentido, podría afirmarse que no hay topología lacaniana que no lo sea del significante $y$, por tanto, que no hay pensamiento lacaniano sin topología (Miller, 2003: 37). De este modo, volviendo al grafo, a través del hiato o la síncopa entre la demanda y la necesidad, en esa misma diferencia, surge la noción fundamental de la primera enseñanza lacaniana: el deseo. Porque si es el Otro quien le entrega al sujeto la forma demandada de su necesidad, el sujeto no hace sino buscar aquel resto inasimilable a la demanda a través 
o por medio del cual, no obstante, puede la demanda llegar a existir, y con ella la necesidad: esta necesidad callada en la demanda, este movimiento de búsqueda hacia un "anterior" previo a todo tiempo verbal es el deseo, una búsqueda más allá del Otro en cuanto lugar en el que se articula la demanda, una demanda de amor, una demanda absoluta (Lacan, 2003: 670). Como señala Darmon, «articulando hacia el Otro su demanda en la cadena significante, demanda condicionada en su inicio por una necesidad, el sujeto encuentra en el Otro una respuesta y un deseo" (Darmon, 2008: 131), por lo que, citando a Lacan, «el sujeto reconoce un deseo más allá de la demanda, un deseo no adulterado por la demanda, lo encuentra, y lo sitúa más allá del primer Otro a quien se dirigía la demanda» (Lacan, 2010: 367). Esto se encuentra representado perfectamente en el grafo del deseo completo:

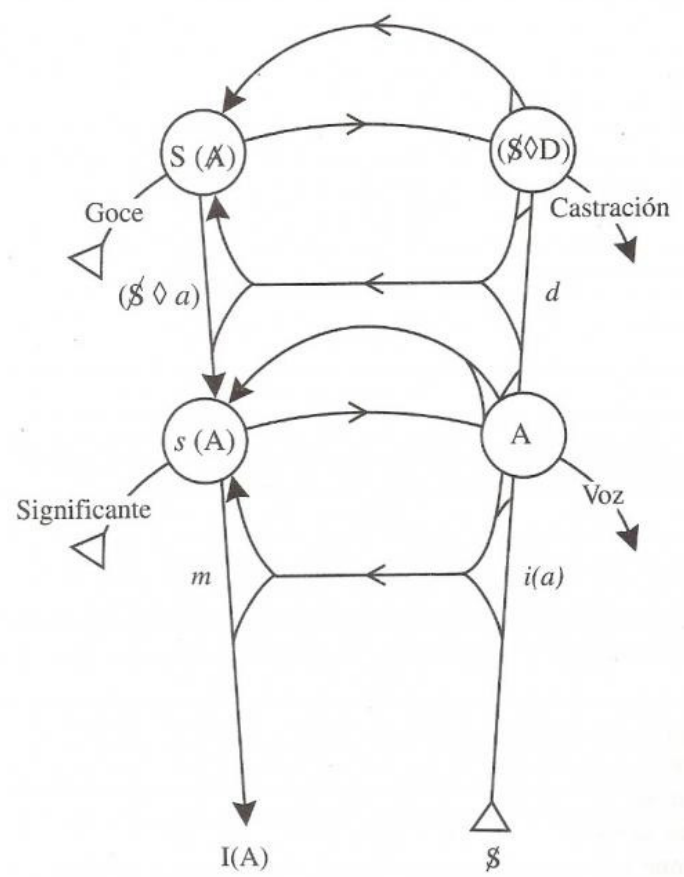

Imagen 2. El grafo del deseo. (Lacan, 2010: 521) 
Este esquema topológico, una vez constituido a través de sus múltiples estadios, consta de dos "pisos». El primero de ellos, el inferior, es aquel compuesto por los vértices $\$, \mathrm{~A}, s(\mathrm{~A})$ e $\mathrm{I}(\mathrm{A})$, no representando sino el movimiento que estructura y conforma la demanda a través de la necesidad. Por otra parte, el piso superior, que Lacan denomina «inconsciente», se figura en el segmento $A-(\$ \triangleright D)-S(A)-s(A)$. El punto al que debemos prestar atención en este momento es el que mantiene en conexión A y $\$ \diamond \mathrm{D}$, esto es, $d$, el deseo. Porque si el Otro no puede satisfacer la necesidad del sujeto más que a través de la demanda, es decir, a través del significante, y si lo propio de un significante es encontrarse en relación diferencial y por tanto dinámica con el resto de los significantes (Cfr. Lacan, 2015: 21), esta demanda no en nunca «en sí», su "para sí» es siempre "para otro». De este modo, como señala Darmon en su estudio topológico del grafo del deseo, $\$ \diamond \mathrm{D}$ no viene a señalar sino que el sujeto constituido en la cadena significante (esto es, \$), atravesando al Otro (A) con su demanda, siendo esta demanda la manifestación de la insatisfacción de la necesidad, busca a través del deseo formarse precisamente a través de esa demanda (D), busca identificarse sin resto con su demanda. Pero siendo el deseo, en cuanto mediación del significante, el deseo del Otro (Cfr. Lacan, 2003: 257), y puesto que la demanda es tal en el significante, aquello que busca el deseo no es sino el significante del Otro, es decir, ese significante que puede significar la cadena significante y como tal significar sin resto su necesidad en cuanto demanda. De lo que se trata en el deseo, por tanto, es de significar al Otro como Otro, en hacer del Otro un significante que pueda traer en plenitud a ese mismo Otro.

Este significante que significa la cadena significante misma, este significante del Otro es, como se sabe, lo que Lacan denomina "falo", afirmando por ello que «el falo no es otra cosa que el significante del deseo del deseo. El deseo no tiene otro objeto que el significante de su reconocimiento» (Lacan, 2015: 530). En este sentido, como señala Nasio, el falo no significa sino el significante en su movimiento, es decir, el deseo (Nasio, 2007: 86) o, como afirma Lacan, «el significante del significado en general» (Lacan, 2010: 248). En este sentido, podríamos representar el significante del significante del siguiente modo: 


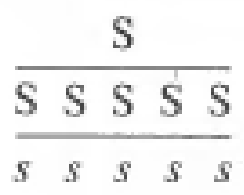

Imagen 3. El significante fálico en la cadena significante.

Ahora bien, como destaca Darmon, «toda la verdad no puede ser dicha puesto que cada significante remite a otro, hay una represión primordial que no puede pasar por el decir algo. Es lo que simboliza $S(\AA)$, el significante de la falta del Otro. Lo simbólico está abierto, la Falta la A barrada» (Darmon, 2008: 175). Por eso mismo, podría representarse gráficamente el movimiento del significante fálico en cuanto S(A) de este modo:

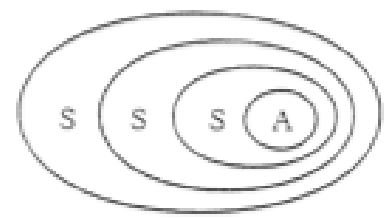

Imagen 4. El significante buscando al Otro.

El significante quiere al Otro, quiere ser del Otro. No obstante, el significante del Otro no deja por ello de ser él mismo un significante, es decir, se encuentra en la cadena significante a la cual quiere significar. De este modo, el significante fálico se situaría en el límite, en un lugar éxtimo, en un «afuera» que permanecería, sin embargo, dentro. Así, puede describirse este significante como el significante de la falta, o el significante que falta en cuanto totalidad no-diferencial de la cadena significante: «el significante fuera del sistema» (Lacan, 2008a: 392), el no-todo, el significante privilegiado que, sin embargo, permanece siempre velado (Lacan, 2008b: 278). Por ello mismo, se comprueba ahora cómo desde $d$, habiéndose el sujeto constituido a través de la demanda $(\$ \diamond D)$ se obtiene $S(A)$ :

La A mayúscula tachada significa lo siguiente: en A -que no es un ser, sino el lugar de la palabra, donde yace, en forma desplegada, el conjunto del sistema de los significantes, es decir, de un lenguaje- falta algo. Lo que allí falta no puede ser más que un significante; por eso la S. El significante que falta en el nivel del Otro: tal es la 
fórmula que da su valor más radical al $S(\AA)$. Ése es, si me permiten, el gran secreto del psicoanálisis. El gran secreto es: no hay Otro del Otro (Lacan, 2015: 331).

En este mismo sentido, señala Darmon, el matema $S(\mathbb{A})$ hace señas hacia un significante que no existe, un significante que falta, puesto que no puede haber Otro del Otro (Darmon, 2008: 135). Así, resulta acertada la afirmación de Miller de que «el deseo es en sí mismo insatisfacción», el «no es eso» perpetuo (Miller, 2009: 204), por lo que debemos entender « $\mathbb{A}$ como aquello que constituye al sujeto como inconsciente, el Otro en la medida en que no es posible significarlo, hacerlo presente: esto es, el Otro como deseo" (Miller, 2013: 20). Pero que A no pueda ser significado no hace de ello, por el contrario, algo insignificante, sino que se constituye como la "estructura no significante de la falta» (Miller, 2013: 34), es decir, una dimensión que se mantiene presente precisamente en cuanto ausencia, en el proceso que Lacan, en otro contexto, describiría como "no deja de no escribirse» (Lacan, 1998: 114). Por ello mismo, como señala Miller, «si no hay Otro del Otro con mayúscula, hay otro del Otro con minúscula» (Miller, 2011: 46). Esto nos conduce, por tanto, a la última conexión que queda por tratar en el grafo del deseo, es decir, aquella que conduce de $S(\AA)$ a s $(\mathrm{A})$ a través de $\$ \triangleright$ a. Esto sitúa la reflexión en el siguiente plano:

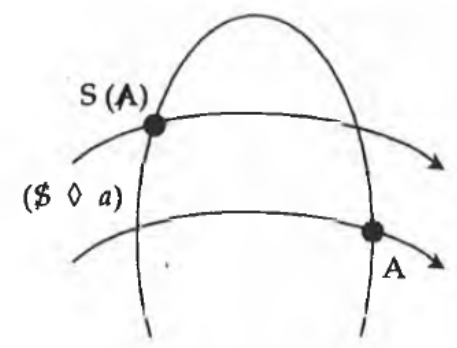

Imagen 5. Sección del grafo del Deseo. (Miller, 1998: 46).

No hay, en definitiva, significante del deseo, significante del Otro, sino "palabrería» (Lacan, 2010: 391). Lo que hay en su lugar, sin embargo, es $\$ \triangleright a$, el matema correspondiente al fantasma. De este modo, el fantasma viene a significar que el sujeto, $\$$, al no poder alcanzar nunca $S(A)$, es decir, al tener que enfrentarse con $S(\AA)$, tan sólo es capaz de acceder a $a$, a un otro, pero no al Otro, por lo que, como señala Lacan, el fantasma se encarga de producir cierta acomodación entre el sujeto y un objeto que 
haría las veces de $\mathrm{A}$, es decir, que «con ayuda de esta relación fantasmática es como el hombre se orienta y sitúa su deseo. De ahí la importancia de los fantasmas» (Lacan, 2010: 450-451) . El fantasma, por tanto, encarna la imposibilidad en $a$, en un objeto (Lacan, 2007a: 138), ya que como afirma Lacan en el seminario $\mathrm{X}$, «desear al Otro, A mayúscula, nunca es más que desear $a »$ (Lacan, 2007a: 194). En definitiva, como señala Palombi, de lo que se trata es de proceder a través de una serie infinita de identificaciones, de $a_{\mathrm{n}}$, hacia A (Palombi, 2009: 356), puesto que el fantasma, tal y como sugiere Miller, «es como una máquina para transformar el goce en placer» (Miller, 2007: 20) ${ }^{1}$. Falo, por tanto, y a son lo mismo, por lo que, como señala Miller, podríamos considerar que en el siguiente esquema

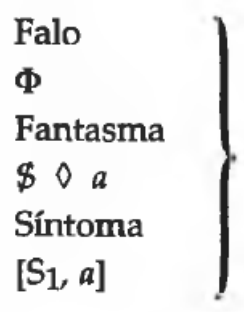

Imagen 6. Sinonimia lacaniana. (Miller, 1998: 85).

todos los términos referirían a una misma dimensión: lo imposible en la cadena significante (Miller, 2011: 85). Así, este objeto a por medio del cual el sujeto da cuerpo a su deseo y podría mantenerlo siempre activo en la medida en que se encuentra siempre insatisfecho cerraría la dinámica del grafo del deseo relacionando el piso superior con el piso inferior: a través de $\$ \diamond$ a se consigue que $S(\mathbb{A})$ entregue $s(\mathrm{~A})$ y, en última instancia $\mathrm{I}(\mathrm{A})$. Toda esta primera topologería lacaniana, por tanto, no es sino la dinámica del fracaso en cuanto imposibilidad de la presencia, es decir, una topología

1 La cuestión del goce escapa los objetivos de este trabajo. Podría indicarse, no obstante, como mero recorrido fugaz por el tratamiento lacaniano, que el goce es lo imposible, ya que el goce sería acceder a S(A). No obstante, si el sujeto pudiese significar al Otro, su misma realidad de sujeto en la cadena significante se disolvería, lo que supondría su muerte. (Cfr. Lacan, 2015: 368). El deseo debe permanecer insatisfecho con vistas a garantizar la existencia del sujeto. Por eso mismo, siendo $a$ el acceso a $S(A)$, la proximidad con este objeto produce la angustia (Cfr. Lacan, 2007a: 98). De este modo, el goce es sacrificado siempre con vistas a obtener un placer, es decir, con vistas a mantener la dialéctica del deseo (Cfr. Lacan, 2007b: 382). 
del vacío, una topología del hueco, del hiato, de la síncopa, pero al mismo tiempo de la continuidad, de la ininterrupción, de la serie.

De lo que se trata ahora es de comprender el modo en que Lacan desarrolla esta topología del vacío en su topologería posterior. Efectivamente, el grafo del deseo supone el primer estadio de la topologería lacaniana y, por ello mismo, no resulta ni la más precisa ni la más intuitiva de sus expresiones. En este sentido, podría afirmarse que las cuatro figuras que van a ser estudiadas a continuación no hacen sino desarrollar, profundizar y aclarar algunos aspectos que ya se encontraban en el grafo del deseo, consiguiendo precisamente a través de este desarrollo expandir la teoría lacaniana hacia algunos aspectos que el grafo, por sí mismo, no era capaz de alcanzar.

\section{La Cinta de Moebius, el toro, la botella de \\ KLEIN Y EL CROSS-CAP.}

Esta segunda etapa de la topologería lacanina comienza en torno al Seminario IX, La identificación, donde Lacan, antes de presentar la primera de sus figuras, el toro, realiza una interesante observación: «a partir de hoy, ustedes lo ven, abro deliberadamente "la era de los presentimientos"” (Lacan, S.F.: 317). En este sentido, Lacan parece venir a indicar que el grafo del deseo pierde el protagonismo que había tenido en seminarios anteriores en favor de un estudio de los cuerpos y de los nudos, siendo el primero de los que escoge el toro. No obstante, por una cuestión de claridad expositiva, se opta aquí por no seguir el orden argumentativo que lleva a cabo Lacan, por lo que la primera de las figuras que se va a desarrollar es la famosa cinta o banda de Moebius.

Señala Lacan que este primer objeto básico no es sino una versión primitiva de la figura compleja que se tratará en último lugar, la esfera provista de un cross-cap. Como explica él mismo, «se fabrica con una banda de papel en la que pegamos las dos extremidades tras haberlas torcido, de manera que el ser infinitamente plano que allí se pasee puede seguirlo sin franquear jamás un borde» (Lacan, S. F: 384). Es decir, la cinta de Moebius lo que viene a señalar es que podemos pasar de la cara interior a la cara exterior de un objeto sin atravesar frontera, borde o límite alguno, por lo que la distinción fundamental entre «interior» $\mathrm{y}$ «exterior» se desmorona, pierde su privilegio metafísico. De este modo, como señala Lacan, «una 
hormiga que se paseara pasaría de una de las caras aparentes a la otra sin necesidad de atravesar el borde» (Lacan, 2007a: 109).

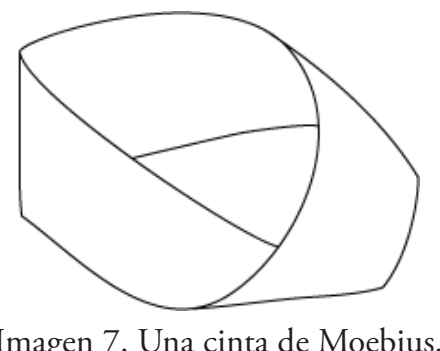

En la dimensión de la clínica lacaniana, como señala acertadamente Nasio, la cinta o banda de Moebius «topologiza» la cuestión fundamental del sujeto dividido y de su decir (Nasio, 2007: 13), esto es, la primera parte del grafo del deseo, en su piso inferior: el diagrama $\$-A$, es decir, la relación necesidad-demanda. No obstante, si bien Lacan no niega la capacidad representativa o explicativa de la banda de Moebius, esto es, la posibilidad de servir como "ejemplo», debe evitarse pensar que la cinta se limita a representar, a exponer o a figurar al sujeto (demanda) como continuidad del interior (necesidad) y el exterior (Otro). Es evidente que, en la topologería lacaniana, «la complejidad reside mayoritariamente en la idea de un sujeto sin interior. Debido a la interdependencia entre sujeto y Otro, el primero está dividido de tal manera que lo más íntimo del sujeto está fuera, y viceversa» (Hoens, 2016: 57). Sin embargo, no se trata únicamente de contemplar la banda de Moebius y a través de su materialidad comprender la naturaleza y dinámica del sujeto lacaniano. Efectivamente, "la topología es el límite sin fronteras, la continuidad sin límites» (Monteiro, 2014: 133), pero requiere actuación, exige el corte, el recorte, el pegar unas partes sobre otras: la experimentación. De este modo, como señala de nuevo Nasio, para comprender adecuadamente la importancia y el significado de la banda de Moebius en la topologería lacaniana debe realizarse un corte a través de la línea media con las tijeras que divida en dos la cinta de Moebius, obteniendo así una banda no moebiusiana. De este modo, citando a Nasio,

no basta entonces con representar el sujeto en el espacio; es menester también el acto de cortar, de trazar una curva cerrada. El acto de decir es del mismo orden porque el significante determina, hiende al sujeto en dos: lo representa y, representándolo, lo 
hace desaparecer. Es cortando la banda como se puede decir: he aquí el sujeto (Nasio, 2007: 16).

El sujeto en su decir constituye, por tanto, una banda de Moebius, pero una de tales características que se disuelve a sí misma, que no permite ser aprehendida de forma fija. Esto, por otra parte, encaja perfectamente con la enseñanza lacaniana: «(Lacan) no intenta externalizar lo real en figuras, lo que significaría encontrar un buen medio de mostración, sino mostrarlo directamente en su imposibilidad gracias a los impasses de la formalización» (Couto, 2018: 231), es decir, que no se trata tanto de ver lo real a través de lo imaginario, sino de destruir lo imaginario para así ver lo real imposible (Couto, 2013: 154), algo que no puede relacionarse sino con la experiencia del «semblante» en la teoría lacaniana. Entendiendo la topologería de este modo, es posible pasar al análisis de la segunda figura: el toro. Ésta nos sitúa de forma inmediata en la dinámica y cinética del deseo y del objeto $a$. Así, si ya se indicó anteriormente que el deseo ( $d$ en el grafo) pretende $S(A)$ pero no puede sino aproximarse a $S(\mathbb{A})$ realizándose a través de $\$ \triangleright a$, esto no viene sino a indicar que el deseo da vueltas en torno a un vacío esencial y necesario y que, por tanto, termina donde comienza, es decir, siempre comienza en el lugar en el que asimismo termina: el deseo nunca finaliza, es repetición y creación de sí mismo a través de esa misma repetición, una «errancia», como la denomina Lacan (Lacan, 2010: 346). Recuérdese, en este sentido, que la figura básica de la dialéctica entre necesidad y demanda se podría representar de este modo:

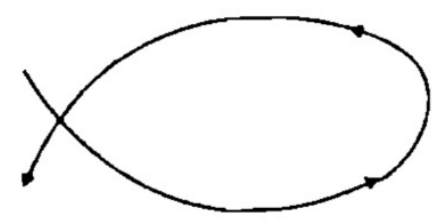

Imagen 8. La demanda. (Nasio, 2007: 14)

Así, si el sujeto reclama la satisfacción de una necesidad al Otro y ésta le es devuelta en forma de demanda constituida que el sujeto asume como propia, produciéndose así la dialéctica interminable del deseo, a esta figura en forma de "pez», tanto en su comienzo como en su final, debería añadírsele un segundo pez, y un tercero, hasta un número imposible de definir. Al multiplicar este dibujo un número $n$ de veces lo que se obtiene 
es la unión de $n$ ocho interiores girando alrededor de un vacío, lo que también podríamos entender como el "plus de goce» (Lacan, 2008a: 2021). De este modo, obtenemos el toro que topologiza el deseo (Cfr. Lacan, S.F.: 325; 386):

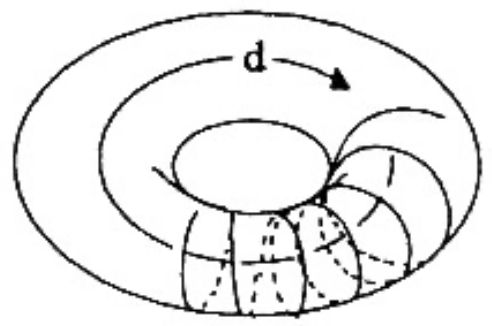

Imagen 9. El toro y la demanda. (Nasio, 2007: 14).

No obstante, señala Lacan, y esto es algo en lo que Darmon insiste, puesto que el vacío no puede colmarse, debe ser suturado por otro toro, un toro complementario que, a su vez, vería su agujero empleado por un tercer toro, y así en número infinito (Lacan, S. F.: 348):

Siendo diferente de sí mismo el significante implica un espacio de diferencia que no puede ser colmado; y el más simple de los significantes, cerrándose sobre sí mismo, no puede sino desdoblarse entre sí mismo y el otro que él es para sí mismo. El toro implica siempre un toro complementario al que se encuentra acoplado. El agujero periférico de uno es el agujero central del otro (Darmon, 2008: 156).

Por lo que la más acertada representación del toro debe ser esta:

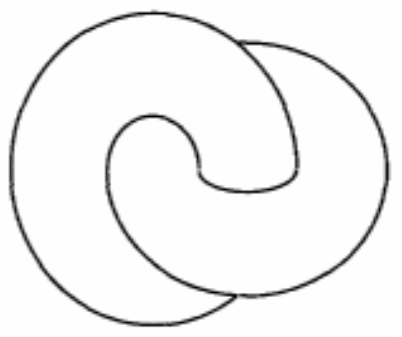

Imagen 10. Segunda imagen del toro. 
Si con la banda de Moebius la topologería lacaniana se situaba en lo que en el grafo del deseo corresponde al segmento $\$$-A y el toro nos permite avanzar en la dinámica $\$-A-d-S \diamond D$, la tercera de las figura permite completar el diagrama $\$-\mathrm{A}-d-\mathrm{S} \diamond \mathrm{D}-\mathrm{S}(\mathbb{A})$, pudiendo realizar la totalidad del grafo únicamente cuando se alcance a desarrollar la esfera provista de un cross-cap. De este modo, se planteaba en el epígrafe anterior la necesidad de que un significante, con vistas a unificar la cadena significante, es decir, de significar al Otro, debía situarse al mismo tiempo como un significante más en la cadena, constituyendo así un significante privilegiado que se erguiría en la dignidad de la Cosa. Esto, por otra parte, condujo a introducir la noción de «extimidad» como posibilidad de concebir un significante actuando desde el límite, quebrando así la oposición entre el dentro y el afuera. Esta mecánica, la disolución del borde en cuanto consolidación del mismo es precisamente la que Lacan topologiza a través de la botella de Klein. Esta figura, sorprendentemente, no es estudiada en el Seminario IX, sino que la presenta Lacan en el seminario dedicado a la angustia y no vuelve a ser desarrollada en los seminarios posteriores.

En el botella o jarra de Klein, figura que puede constituirse a través de la banda de Moebius², el borde, aquello a partir de lo cual la jarra se comunica con el afuera y que le permite albergar el interior (por más que éste sea, propiamente, un vacío), no existe en absoluto, sino que la botella se cierra sobre sí misma: escancia su contenido, su interior, hacia sí misma. En este sentido, esa figura imposible del borde se comprende como $S_{1}$, esto es, como aquél significante que significa la cadena, situándose por tanto más allá de ella, pero al mismo tiempo siendo un significante entre otros, un significante más del Otro.

2 Así, siguiendo las animaciones de Jos Leys (disponible en http://www.josleys.com/), se muestra que:
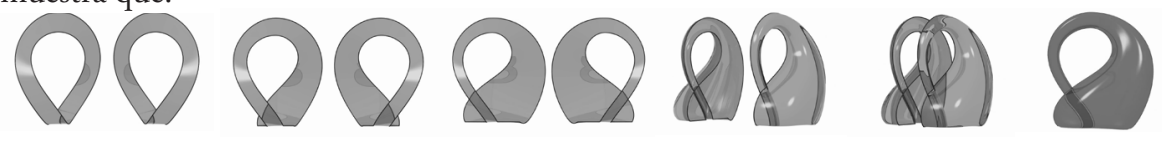


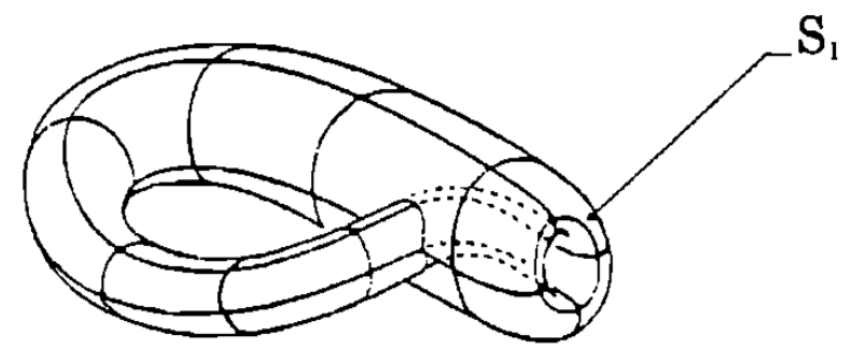

Imagen 11. La botella y el significante. (Nasio, 2007: 18).

El falo se situaría, por tanto, en el punto $S_{1}$, ni dentro ni fuera, en lo que Derrida denominaría «indecidibilidad». Se llega de este modo al último de los cuerpos topológicos lacanianos, la esfera provista de un crosscap, el quizá más extenso y complejo de los objetos estudiados por Lacan y que sintetiza el grafo del deseo en su dinámica completa, implicando por tanto las figuras anteriormente desarrolladas. En este sentido, ya se indicó que Lacan consideraba que la esfera provista de un cross-cap no era sino una figura equivalente a la cinta de Moebius. Ahora bien, el término «equivalente» se emplea aquí en un sentido estrictamente topológico: «dos espacios topológicos son equivalentes cuando entre ellos se puede definir un homeomorfismo; desde un punto de vista intuitivo, esto quiere decir que se puede deformar cualquiera de ellos para obtener el otro» (Amster, 2010: 39). Sin embargo, la construcción de la esfera provista de un cross-cap desde una perspectiva matemática resulta un proceso extenso que requiere comprender las nociones de "homomorfismo», «inyección/inmersión», «plano proyectivo/ordinario». En este sentido, el objetivo del presente texto queda lejos de alcanzar el detalle y cuidado en la exposición de la construcción que han llevado a cabo Nasio y Amster en sus obras. De lo que se trata, por el contrario, es de comprender cómo la esfera provista de un cross-cap sintetiza el grafo del deseo, poniendo en cuestión la oposición dentro/afuera (cinta de Moebius y botella de Klein), la escisión del sujeto (cinta de Moebius) y su dinámica en el deseo (toro) como objeto $a$. De este modo, si la botella de Klein permite situar el estudio topológico en $S(\mathbb{A})$, se atraviesa con la esfera provista de un cross-cap el fantasma $(\$ \triangleright a)$ y concluye la primera parte de la topologería lacaniana. 
Como se ha indicado anteriormente, la esfera provista de un cross-cap se constituye a través de la cinta de Moebius ${ }^{3}$. De este modo, partiendo de la banda moebiusiana (figura 12) y realizando un corte a la cinta (figura 13) se obtiene un cuerpo similar a un túnel (figura 13). Desde aquí, por equivalencia, se puede curvar el cuerpo de la figura hasta obtener una semiesfera (figuras 15, 16 y 17), doblándose ésta sobre sí misma y realizando una torsión (figura 18) que permita unir sus extremos (figuras 19 y 20). De este modo, se obtiene el cross-cap (figura 21), al que debe añadírsele un disco para obtener la figura definitiva de la esfera provista de un cross-cap (figura 22):

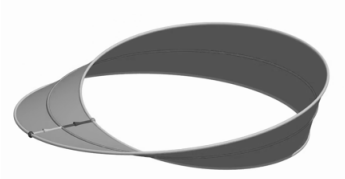

Figura 12

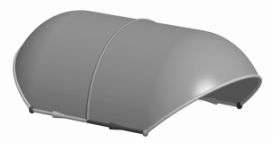

Figura 15

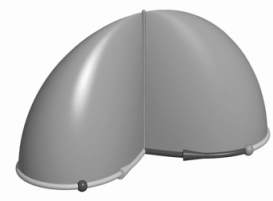

Figura 18

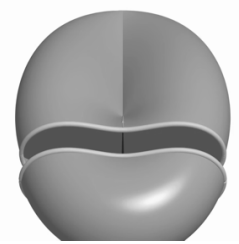

Figura 21

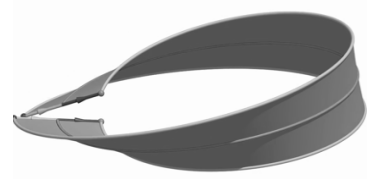

Figura 13

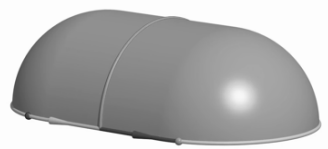

Figura 16

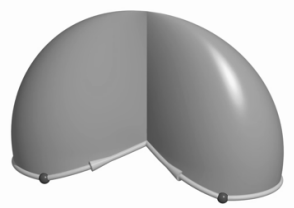

Figura 19

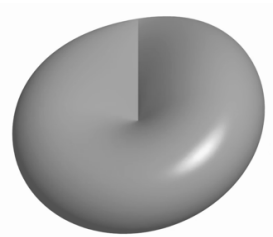

Figura 22

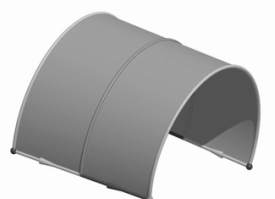

Figura 14

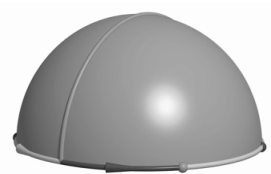

Figura 17

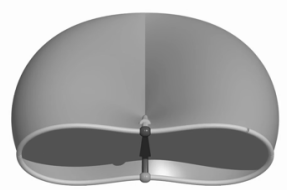

Figura 20

3 A lo largo de esta exposición se hace uso de las ilustraciones de Jos Leys. 
Lo interesante de esta figura es que permite reunir la cinta de Moebius con el disco, que aquí representa el objeto $a$. Como señala Nasio, al efectuar un corte sobre el cross-cap ya constituido lo que comprobamos es el ocho interior que reúne el objeto a y la cinta de Moebius (Lacan, 2007a: 110): aun siendo objetos heterogéneos, siempre han permanecido unidos (Nasio, 2007: 75). De este modo, si bien la representación gráfica anterior (lo que Nasio denomiba el cross-cap concreto, imperfecto) sirve para comprender la constitución de la esfera provista de un cross-cap, no deja de ser equivocada, siendo el hecho propiamente relevante concebir que a través del ocho interior (sujeto) (Miller, 2003: 43) y de la repetición se aúna la cinta de Moebius, el objeto a en cuanto residuo (disco) y la esfera provista de un cross-cap (Lacan, S. F.: 668) (lo que se denomina el cross-cap abstracto):

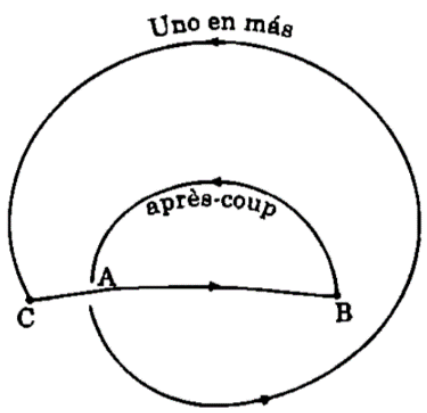

Imagen 23. El cross-cap. (Nasio, 2007: 79).

De este modo, únicamente a través de la esfera provista de un crosscap en cuanto cuerpo topológico puede comprenderse la relación entre la constitución del sujeto a través de la repetición y del deseo y su íntima y necesaria unión con el objeto $a$. Esto no viene sino a señalar, por tanto, que en la topologería lacaniana la esfera provista de un cross-cap, y los cortes a ésta realizada, sintetizan de forma precisa la dinámica del grafo del deseo que había servido a Lacan como primer intento en su objetivo de topologizar la práctica psicoanalítica. No obstante, Lacan abandona paulatinamente estos objetos en favor de los nudos. De este modo, no se comparte aquí la clasificación realizada por Miller de los períodos de la enseńanza Lacaniana, donde propone un "período topológico» representado por el toro y el nudo borromeo sino que, a nuestro juicio, 
el período dedicado a los nudos constituyen una unidad en sí mismo y, por tanto, se encuentra perfectamente diferenciado, tanto en tiempo como en tono, del período estrictamente topológico, siendo no obstante ambos estadios de la topologería lacaniana.

\section{LOS NUDOS Y LA DISOLUCIÓN DEL PSICOANÁLISIS.}

El momento en el que Lacan introduce su topología de los nudos se corresponde al progresivo desplazamiento lacaniano desde la dialéctica del deseo hacia el goce de lo real. Así, si bien es cierto que la noción de «goce» se encuentra ya presente en la primera enseñanza lacaniana, no es sino hasta el seminario X cuando el objeto $a$, y con él la dialéctica del deseo y la dinámica del semblante se relacionan de forma directa con la imposibilidad e interdicción del goce. En este sentido, la última enseñanza lacaniana se orienta hacia el estudio de cómo el «plus de goce» actúa de tal modo que permite mantener activa la cinética psíquica a través de las operaciones fantasmáticas de los objetos a. Tal y como señala Lacan, «el plus-de-gozar es función de la renuncia al goce por el efecto del discurso. Eso es lo que da su lugar al objeto a» (Lacan, 2008a: 18). En este sentido, si bien la enseńanza lacaniana hasta este momento y la demanda, es a través de la noción de angustia con lo que Lacan comienza a dar protagonismo a la dimensión no-lingüística en el discurso, esto es, al goce en el deseo: lo real. Así, al señalar Lacan que la distinción entre real y semblante podría parecer que su objeto de estudio va a ser lo real como algo radicalmente distinto del semblante. No obstante, y esto es algo en lo que Lacan insiste, no hay que separar semblante y real, deseo y goce, como si fuesen dos instancias que pudiesen aprehenderse de forma independiente: desde el momento en el que sólo hay "parecer» (parêtre) nunca podremos estudiar lo real de forma independiente, sino siempre imbricado con lo simbólico y lo imaginario. En este sentido, más que de sentido (deseo, verdad) y goce (real), lo que debería tratarse en la topologería es el «joui-sens» (Miller, 1998: 293), el «gocentido» que reúne aporética pero indisolublemente las tres dimensiones psíquicas: real, simbólico e imaginario.

Así, resaltando la indisolubilidad semblante-real, afirma Lacan que «todo lo que es discurso sólo puede presentarse como semblante, y nada se construye allí sino sobre la base de lo que se llama significante. Desde la perspectiva que les presento hoy, el significante es idéntico al estatuto 
como tal del semblante» (Lacan, 2009: 13), es decir, que «el goce sólo se interpela, se evoca, acosa o elabora a partir de un semblante» (Lacan, 2009: 112). De este modo, Lacan rechaza cualquier posibilidad de aprehender lo real por sí mismo, esto es, sin mediación del semblante: “¿qué puedo saber? Respuesta: nada que no tenga la estructura del lenguaje en todo caso» (Lacan, 2012: 561). La topologería, por tanto, tiene que tratar con la dimensión del semblante, esto es, con lo simbólico aproximándose hacia lo real y al mismo tiempo limitándolo. En este sentido, una primera aproximación topológica a este nuevo dispositivo psíquico la realiza Lacan en el seminario XX, donde introduce el siguiente esquema:

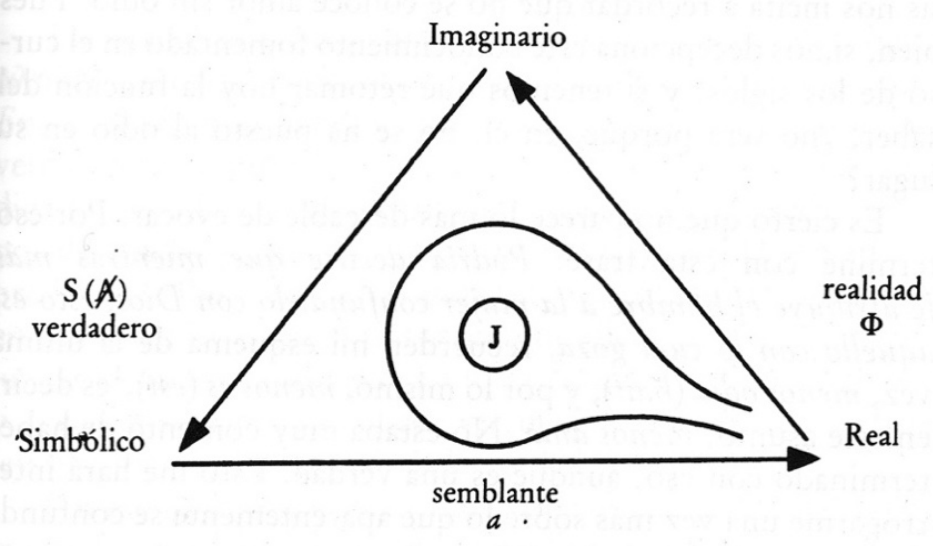

Imagen 24. El Goce. (Lacan, 1998: 109).

Este esquema consta de tres direcciones: lo imaginario dirigiéndose a lo simbólico, lo simbólico intentando apresar lo real y lo real en su conexión con lo imaginario. Se observa, por otra parte, que Lacan sitúa el semblante y el objeto $a$ en el vector S-R, no extendiéndolo a la totalidad de su esquema. En este sentido, la relación entre lo real, lo simbólico y lo imaginario se condensa y corrige sus inexactitudes expositivas en la topologería lacaniana a través del estudio del nudo borromeo. Tal y como señala Darmon, «el nudo borromeo, cuya elaboración y cuestionamiento ocuparon un espacio central en los últimos años de la obra de Lacan, es la solución topológica al problema siguiente: ¡cómo es que las dimensiones, distintas e independientes si se las considera de a dos, de lo Simbólico y lo Imaginario y de lo Real, se mantienen sin embargo unidad?» (Darmon, 
2008: 27). Resulta evidente, por tanto, que el nudo borromeo viene a responder a la cuestión que había ocupado a la topología lacaniana desde el grafo del deseo, por lo que es posible considerar este nudo como una versión sintetizada y plegada del primer estadio de la topologería lacaniana, es decir, una recapitulación del grafo y los cuerpos topológicos. Así, cada uno de los anillos que constituyen el nudo se corresponde con una de las tres dimensiones:

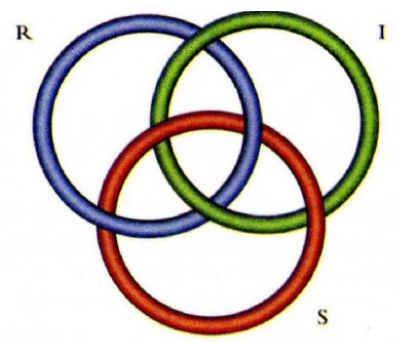

Imagen 25. El nudo borromeo. (Lacan, 2006: 20).

La particularidad de este nudo es perfectamente explicada por Amster en su estudio topológico de Lacan: «si denotamos con el signo > la propiedad de un redondel de pasar sobre otro, tenemos entonces tres redondeles $(\mathrm{R}$, S, I) con la siguiente particularidad: $\mathrm{R}>\mathrm{S}>\mathrm{I}>\mathrm{R}$ » (Amster, 2010: 135), lo que no es, como él mismo señala, sino cierta versión del juego "piedra, papel y tijera». De este modo, el nudo borromeo sintetiza las nociones de objeto $a$, semblante y deseo, en lo que Lacan denomina «esquema RSI»:

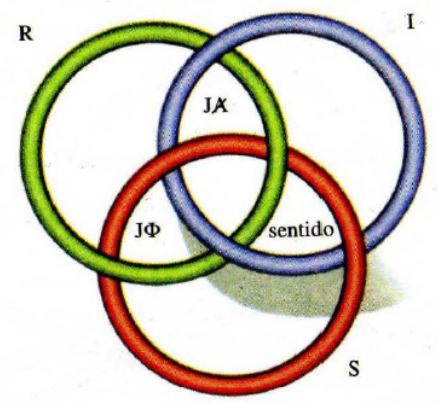

Imagen 26. Esquema RSI. (Lacan, 2006: 48).

Ninguno de los aros tiene preeminencia sobre el resto, ni siquiera lo real sobre lo simbólico, por lo que si se corta uno, el nudo se deshace. Con esto 
Lacan no viene sino a topologizar un hecho fundamental de su enseñanza: el frágil equilibrio de lo psíquico en cuanto dinámica interminable entre lo real, lo simbólico y lo imaginario. Ahora bien, cuando esta unión se quiebra, cuando alguno de los aros falla en su solidaridad y el nudo se deshace, Lacan introduce la noción de "sinthome» como aquel aro suplementario que vendría a reunifica el punto de quiebra en el nudo, permitiendo así la supervivencia del sujeto.

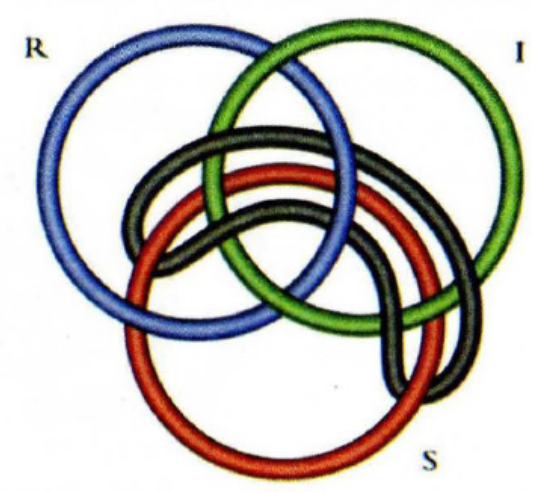

Imagen 27. El sinthome. (Lacan, 2006: 21).

El sinthome podría comprenderse, por tanto, casi como el objeto $a$, en el sentido de que permite a la dinámica psíquica garantizar su supervivencia a través de un nudo de más, a través de una nueva vuelta. En este sentido, el sinthome realiza el verdadero deseo del goce: no realizarse, no disolverse. Como se indicó anteriormente, si el sujeto se acercaba en exceso al falo, al objeto $a$, correría el riesgo de satisfacer su deseo y, con él, su misma existencia, siendo ésta la enseñanza fundamental del grafo del deseo y de los cuerpos topológicos posteriores. De este modo, se comprueba cómo el nudo borromeo realiza el paso definitivo en la topologería lacaniana: el vacío y su movimiento es comprendido ahora desde la trabazón del nudo borromeo, y la significación del falo, del Nombre del Padre y del objeto $a$ no vienen a ser sino otro nudo que garantiza la estabilidad y la permanencia del borromeo. Así, el grafo del deseo, primitivo en la medida en que no permitía comprobar las tres dimensiones psíquicas simultáneamente en su vinculación esencial, halla su perfección en el nudo borromeo, sin la necesidad de recurrir a matemas, explicaciones o ejemplificaciones. Es con el nudo, por tanto, como concluye la topologería lacaniana, y casi podría 
decirse que concluye la enseñanza «propiamente» lacaniana, dando lugar posteriormente a lo que Miller denomina el «ultimísimo» Lacan, donde se lleva acabo, efectivamente, una disolución de su enseńanza anterior, incluyendo la topologería (Miller, 2014: 213).

\section{Conclusión.}

A través del recorrido por la topologería se comprueba cómo la totalidad de la enseńanza lacaniana puede ser sintetizada en una serie de figuras y cuerpos. Por otra parte, este estudio permite atender a los diferentes estadios de la teoría lacaniana: un primer momento centrado en la dialéctica del deseo y en la constitución significante del deseo, que encuentra su materialización topológica en el grafo y en los cuatro cuerpos (banda de Moebius, toro, botella de Klein y esfera provista de un cross-cap), pudiendo aquí reconocer alguna de las nociones fundamentales en la obra de Lacan tales como falo, objeto $a$ o fantasma; y un segundo momento donde la reflexión lacaniana vira hacia la relación entre el deseo y el goce, introduciéndose así el concepto de «semblante» y de lo «real» imposible en cuanto algo diferente a la «realidad» significante. Esta segunda etapa de la teoría lacaniana se topologiza fundamentalmente a través del nudo borromeo y de su complemento en forma de sinthome.

Así, se concluye no sólo que el pensamiento lacaniano puede ser representado gráficamente con ejemplos ilustrativos provenientes de la topología matemática sino que, por el contrario, el progreso mismo del psicoanálisis lacaniano no podría haberse llevado a cabo si Lacan no se hubiese visto obligado a recurrir a la topología matemática. En este sentido, la topología no constituye simplemente una herramienta útil para el psicoanálisis lacaniano, un feliz encuentro, sino que éste se muestra esencial y necesariamente como topologería.

\section{REFERENCIAS BIBLIOGRÁFICAS}

Amster, P. (2010): Apuntes matemáticos para leer a Lacan. Buenos Aires: Letra Viva.

Cao, J. (S.F.): «Mathematizing the Limits of Time: Heidegger, Derrida and the Topology of Temporality», Math.HO (arXiv:1910.05359). 
Couto Triska, V. H. y Leao D’Agord, M. R. (2013): «A topologia estrutural de Lacan», Psicologia Clinica 25-1, pp. 145-161.

Couto Triska, V. H. y Leao D’Agord, M. R. (2018): «The scientific roots of Lacan's structural topology", Ágora 2 mayo/agosto, pp. 224-232.

Darmon, M. (2008): Ensayos acerca de la topología lacaniana. Buenos Aires: Letra Viva.

Hoens, D. (2016) «Why Topology?» en M. Friedman y S. Tomsic (ed.), Psychoanalisis: Topological Perspectives. Bielefeld: Transcript.

Lacan, J. (1998): El Seminario XX. Aun. Buenos Aires: Paidós.

Lacan, J. (2003): Escritos. Buenos Aires: Siglo XXI.

Lacan, J. (2006): El Seminario XXIII. El Sinthome. Buenos Aires: Paidós.

Lacan, J. (2007a): El Seminario X. La angustia. Buenos Aires: Paidós.

Lacan, J. (2007b): El Seminario VII. La ética del psicoanálisis. Buenos Aires: Paidós.

Lacan, J. (2008a): El Seminario XVI. De un Otro al otro. Buenos Aires: Paidós.

Lacan, J. (2008b): El Seminario VIII. La transferencia. Buenos Aires: Paidós.

Lacan, J. (2009): El Seminario XVIII. De un discurso que no fuera del semblante. Buenos Aires: Paidós.

Lacan, J. (2010): El Seminario V. Las formaciones del inconsciente. Buenos Aires: Paidós.

Lacan, J. (2012): Otros escritos. Buenos Aires: Paidós.

Lacan, J. (2015): El Seminario VI. El deseo y su interpretación. Buenos Aires: Paidós.

Lacan, J. (S. F.) El Seminario IX. La identificación. Edición Crítica de Ricardo E. Rodríguez Ponte.

Miller, J.-A. (1998): Los signos del goce. Buenos Aires: Paidós.

Miller, J.-A. (2003): «Mathemes: Topology in the Teaching of Lacan» en E. Ragland y D. Milovanovic (ed.), Lacan: Topologically speaking. New York: Other Press, pp. 28-48.

Miller, J.-A. (2007): Dos dimensiones clinicas: sintoma y fantasma. Buenos Aires: Manantial.

Miller, J.-A. (2009): De la naturaleza de los semblantes. Buenos Aires: Paidós. 
Miller, J.-A. (2011): La experiencia de lo real en la cura psicoanalitica. Buenos Aires: Paidós.

Miller, J.-A. (2013): La angustia lacaniana. Buenos Aires: Paidós.

Miller, J.-A. (2014): El ultimísimo Lacan. Buenos Aires: Paidós.

Monteiro, M. P. (2014): «A topologia de Lacan», Estudos de Psicanálise 41, pp. 133-140.

Nasio, J.-D. (2007): Topologería. Buenos Aires: Amorrortu Editores.

Palombi, F. (2009): "Neither inside nor outside. Consideration on the structure of the subject and of language in Jacques Lacan», Ethics \& Politics XI-1, pp. 351-360.

Pablo Bernardo Sánchez Gómez es Investigador Pre-Doctoral FPI UNED, donde desarrolla su trabajo en torno a la relación entre Martin Heidegger y Jacques Derrida.

Lineas de investigación:

- Pensamiento heideggeriano, deconstrucción, psicoanálisis lacaniano.

\section{Publicaciones recientes:}

- «La topología derridiana como lugar de lo imposible». Anales del Seminario de Historia de la Filosofía, vol. 38. núm. 2 (2021). pp. 347-358.

- «La traducción entre Heidegger y Derrida». Pensamiento, vol. 76. núm. 289 (2020). pp. 371-389.

- «La distancíia imposible entre el 'cap' derridiano y la 'polis' heideggeriana». Isegoría, núm. 62 (2020). pp. 207-226.

- «La angustia no es sin objeto: Lacan lector de Heidegger». Agora, Papeles de Filosofía, vol. 39. núm. 1 (2020). pp. 141-161.

Dirección electrónica: psanchez@fsof.uned.es 